\title{
Wound Infection Sensing Method and Markers
}

\author{
Shenyi Qian ${ }^{1}$ Jie Ren ${ }^{1}$, Haohan Ning ${ }^{1}$, Kunpeng $\operatorname{Ren}^{1}$, Tong Sun $^{2}$ \\ ${ }^{1}$ School of Computer and Communication Engineering, Zhou University of Light Industry, \\ Zhengzhou, Henan, Chinese \\ ${ }^{2}$ College of Information and Management Sciences, Henan Agricultural University, \\ Zhengzhou, Henan, Chinese
}

\begin{abstract}
Wound infection is a serious complication that causes slow wound healing and poses a great risk to the patient. Traditional methods of infection diagnosis include wound clinical description diagnosis and microbiological test diagnosis. However, the clinical description of wound diagnosis is prone to error and the microbiological test is time-consuming. Beyond invasive and costly methods, more reliable alternatives remain scarce. In this paper, the sensing methods and biomarkers of wound infection are discussed. Starting from the detection of wound infection sensor methods, the sensor methods are divided into three categories. The current research progress and marker characteristics of each sensor method were reviewed. The research trend of biomarkers for wound infection evaluation was discussed. For odor sensing, ion migration spectrometry, mass spectrometry and gas chromatography-mass spectrometry provide a rapid, simple and effective new method for the detection of volatile compounds, which has high application value; For taste sensing, it is possible to develop bionic sensors inspired by insect odor receptors in the future. Compared with existing sensors, the sensor has the advantage of a larger range of independent sampling in odor space, and can better identify volatiles. For physical sensing, devices embedded in dressings or site-of-care techniques are utilized to enable continuous, rapid wound assessment and monitoring. In recent years, advances have been made in detection of microorganisms, including the assessment of the diversity of bacterial communities present in wounds and the detailed development of specific markers.
\end{abstract}

Keywords: Wound infection, Sensing methods, Biomarkers.

\section{Introduction}

The etiology of wound is varied: peripheral vascular disease, infectious disease; Secondary trauma, neurological, immune, tumor, coagulation or metabolic diseases, and iatrogenic injury can disrupt tissue repair mechanisms[1-3]. Skin ulcers are a fairly common event, with a current prevalence of $0.18 \%$ to $0.32 \%$ and a prevalence of $0.78 \%$, with a significant increase with the median age of the population[4]. Foot ulcers, for example, occur in about $25 \%$ of people with diabetes and are the leading cause of non-traumatic amputations in developed countries[5]. In the United States, chronic wounds as a whole are estimated to affect about $1-2 \%$ of people throughout their lives[6], That translates to an estimated 6.5 million patients a year at a cost of $\$ 25$ billion. In the United States, chronic wounds as a whole are estimated to affect about $1-2 \%$ of people throughout their lives[7-8]. Under normal physiological conditions, wound healing is a biological process, including hemostasis, inflammation, proliferation and tissue remodeling[9]. Inflammation is the body's adaptive response to physiological and pathological threats, such as trauma, infection, ischemia, poisoning, or autoimmune injury[10-11]. In most areas of biological research, a large number of biomolecules (such as biomarkers) and other physiological markers have been shown to be useful in the diagnosis and treatment of wound infections. Aming these molecular analytes, biomarkers are generally considered to be quantifiable markers that indicate some biological state of the human body. These, biomarker sensors have great potential for early diagnosis and personalized treatment of diseases. Therefore, biomarker sensors have great potential for early diagnosis and personalized treatment of diseases[12-14]. Biomarkers not only give us information about existing diseases, but more importantly, they provide personalized information about underlying medical conditions. By analyzing results between normal samples and patients, this information will provide users with morbidity, subclinical status and other biological information in a rapid manner. Therefore, the detection of biomarkers is of great significance to human health[16]. There are two types of biosensor-bases applications in modern medical diagnosis at present: one is a very accurate and precise detection biosensor, and the other is a low-cost, real-time and on-site sensor device[17]. The former helps us to study the mechanism of disease and is the gold standard to measure the status of biomarkers themselves, while the latter meets the needs of early diagnosis or community screening. Recent research has seen many technological advances in electrochemical sensors and biosensors including novel materials, paper-bases electrodes, microfluidics, integrated sensors, wearable devices, smartphone technology and more[18-22]. At present, there are also reviews on biomarker analysis, but there are few reviews on biomarkers and sensing methods at the same time, especially on wound infection. In recent decades, researchers in various fields have put forward a large number of methods for auxiliary diagnosis of wound infection from different technical routes. In this paper, existing wound infection information sensing methods for biomarkers are classified into three categories, namely: Odor sensing methods, odor sensing methods and physical sensing methods were compared, analyzed and discussed.

\section{Odor Sensing and Its Biomarkers}

Odor sensing measures wound signature odors, which are specific chemicals that evaporate from the wound, to get information about infection. Gc-ms can detect and identify infectious diseases. However, such instruments are large, expensive and cumbersome, and require trained staff to use them. For this reason, many researchers use electronic noses-A device that attempts to mimic a biological olfactory system as a means of detecting these biomarkers. Another technique, ion migration spectroscopy(IMS), is widely used in security applications. These instruments have high sensitivity, low drift and good selectivity levels. For this reason, they have found favor in the field of medical 
diagnosis[23]. Volatile organic compounds (VOCs) are produced by microorganisms, such as those present in infected wounds. Literature[23] describes the use of a device to distinguish VOCs from infected wounds and colonized wounds. These results indicate that it has a good ability to distinguish infected wounds from uninfected wounds. Three common types of wound infection bacteria are Escherichia coli, pseudomonas aeruginosa, and the main volatile metabolites of Staphylococcus aureus. Odor sensing method for wound infection detection has many advantages, such as no invasion, quick response, economical and convenient use, but it also has some disadvantages, such as poor anti-interference ability and difficult quantitative analysis. At present, odor sensing methods are mainly based on electronic nose technology, ion migration spectrometry technology, mass spectrometry and gas chromatography-mass spectrometry technology to carry out research and application exploration.

\subsection{Smell Sensor}

Traditional bacterial detection methods include colony culture and plate counting, biochemical and metabolic tests, ELISA, PCR and so on[24]. Most of these methods have the disadvantage of consuming a lot of manpower, time and equipment, which limits their application. Xu Tingting et al. studied a biosensor in 2019, which has the advantages of high sensitivity, easy miniaturization and detection in complex systems. The content of Staphylococcus aureus in solution can be accurately determined by analyzing the change of electrochemistry signal MGCE caused by the specific binding of Staphylococcus aureus with modified antibody in solution[25]. Considering the importance of early diagnosis and identification of bacteria, Randall A. Allardyce et al described, identified and quantitatively analyzed the headspace VOCs of five clinically important bacteria cultured in medium. In this study, ion-flow mass spectrometry can detect trace volatile metabolites in the headspace of $24 \mathrm{~h}$ conventional blood culture flask[26]. A polymer sensor is being developed to monitor the headspace of metabolites produced by surface wounds and burns[27]. The most common bacterial species in wounds were analyzed by gas chromatography-mass spectrometry (GC-MS) to identify key volatile markers for sensor detection. Successful design and manufacture of suitable sensor arrays can be further tested using PCR techniques to determine their specificity and volatility.

\subsection{Volatile Metabolites as Markers}

Wound-invading bacteria play a vital role in various complex processes of wound healing. The composition of metabolites is specific to the bacteria and their growth stages in the wound. Direct monitoring of volatile molecules at the wound site and detailed analysis of volatile organic compound concentrations above the wound can accurately monitor wound infection. During bacterial valuation, different metabolites are produced, their composition and concentration depending on the species and growth stage of the bacteria. Many of these substances are volatile and often cause the typical wound odor after infection[28].

Many byproducts of bacterial metabolism are airborne and have specific odors, for example, certain anaerobic bacteria produce unpleasant odors. Series gas chromatography-mass spectrometry (GC-MS) is a traditional method for volatile substances analysis, but the instrument is bulky and expensive. A common alternative is to use solid-state gas sensors, which rely on variations in conductivity, capacitance, work function, mass, optics, and reaction energy that may depend on gas-solid interactions[29]. Next-generation sequencing technologies for bacterial identification have the advantages of accuracy, flexibility, parallel processing and ease of automation. It enables high-resolution analysis of the entire wound microbiome, which is important for targeting appropriate therapeutic effects. Because of the short analysis time, this method can be used as an indicator of rapid infection.

\section{Taste Sensing and Its Biomarkers}

Infection is the most common cause affecting wound healing, and the common pathogens of wound infection include Pseudomonas aeruginosa, Escherichia coli, Acinetobacter baumannii, staphylococcus aureus, etc. Timely diagnosis of the bacterial type of wound infection is beneficial to guide the doctor in charge to select appropriate antibiotics, so as to kill the bacteria on wound and promote the wound healing. In fact, the special odor produced by bacteria infected on the wound has already been produced before there is obvious secretion on the wound, and the concentration of volatiles is also different at different stages of bacterial growth[30]. The electronic nose can monitor and identify specific smells with the help of specific odor sensors. According to the difference of gas and concentration in the wound microenvironment between patients and normal people, the disease diagnosis and wound infection monitoring can be realized, and the bacterial types of wound infection can be diagnosed[31].Because of the variety of gases, it is not easy to detect the smell accurately. Like a lot of mature technologies: Alcohol sensor, carbon dioxide sensor, carbon monoxide sensor, is also a kind of smell sensor, but it only detects a single type of gas, and the smell of gas is not our human taste can be recognized. But the principle is the same, as long as we design a gas sensor that can target a variety of smells, we can use the algorithm to determine the smell.

\subsection{Taste Sensing}

Many byproducts of bacterial metabolism are airborne and have special smells that can provide clues to the identity of the bacteria. For example, certain anaerobic bacteria produce unpleasant smells. Volatile organic compounds (VOCs) released by chronic wound injury can be extremely complex[32]. The challenge is to develop sensors that can provide similar information in real time, at lower cost and with better portability. A study[33] solved the problem of using metal oxide sensors in the presence of background gas. A new method based on an array of 15 gas sensors to eliminate background and identify wound infection was proposed for wound infection detection using an electronic nose in mice. Compared with existing sensors, this sensor has the advantage of a larger range of independent sampling in odor space, and can better identify volatiles[34].

\subsection{Cytokines and Inflammatory Factors as Biomarkers}


A series of host biomarkers, including procalcitonin (PCT), C-reactive protein (CRP), human neutrophil apolipoprotein, interleukin-6, have been reported to participate in the pathogenesis and inflammatory regulation mechanism of infection, and play an important role in the differential diagnosis of infection. Bacterial infection, especially severe bacterial infection, is associated with elevated levels of inflammatory factors that persist for a long time, thus aiding the early diagnosis of acute infections.

In general, levels of procalcitonin are extremely low in the serum of healthy people or people who are not infected with bacteria. Literature[35] has shown that serum procalcitonin can be used as a specific indicator for the early diagnosis of systemic severe bacterial infection, so it has relatively high clinical diagnostic and differential value. HNL is an inflammatory indicator that has been discovered in recent years. It has high sensitivity and specificity to distinguish acute infections caused by bacteria or viruses, especially early infections. Up to now, the application of HNL in the differential diagnosis of bacteria and viruses is limited to scientific research experiments and has not been popularized in clinical practice. More data and larger sample studies are needed to confirm the diagnostic performance of HNL in the differential diagnosis of bacteria and viruses. Studies have shown that HNL can increase at 6-8 $\mathrm{h}$ after infection, and the peak time is $18 \mathrm{~h}$ earlier than CRP and PCT, so it can be used in early bacterial infection[36]. As an acute reactive protein, CRP is highly sensitive to infection-induced inflammation, and its levels rise rapidly when inflammation occurs. In general, any infection that causes an inflammatory response in the body raises CRP levels. Thus, CRP levels can be elevated in both bacterial and viral infections.

\subsection{Enzymes as Biomarkers}

The activity of enzymes in the wound directly reflects the intensity and type of immune response. They will play a relevant role in rapid and simple wound monitoring to determine whether a wound is infected. Enzyme-based assays have the advantage of rapid response to changes in enzyme activity levels. This study[37] identified the diagnostic properties of enzymes medulloperoxidase, human neutrophil elastase (HNE), lysozyme and cathepsin-G for detection of wound infection compared with wound swabs. Three promising enzyme models were identified for the detection of wound infection. Compared to wound swabs, enzyme analysis is fast, easy to use and superior to clinical judgment. Human neutrophil elastase (HNE) and cathepsin G (CatG) are involved in the pathogenesis of many inflammatory diseases. Andrea Hasmann et al.[38] showed the potential of HNE and CatG as markers for early detection of infection in this study. Significant differences in HNE and CatG levels were observed in infected and uninfected wound fluids, with infected wound fluids leading to substrate hydrolysis significantly higher than uninfected wound fluids.

\section{Physical Sensing and Its Biomarkers}

Physical sensing method through image, spectrum, temperature and other physical signals to measure wound infection physiological indicators, such as wound size, temperature, color, $\mathrm{pH}$, wound tissue pressure, etc. Such methods usually include Near Infrared Spectroscopy, Image sensor, Pressure sensor, Temperature sensor and so on. Tim R. Dargaville et al.[39] discussed recent advances in detecting markers associated with wound healing and infection, using devices embedded in dressings as site-of-care techniques to enable sustained or rapid wound assessment and monitoring. Methods include direct or indirect detection of bacteria using biological and chemical sensors of wound exudates and volatiles, and monitoring $\mathrm{pH}$, temperature, oxygen, and enzymes. Spectral and imaging techniques as advanced wound monitoring techniques are reviewed in this paper.

\subsection{Physical Sensor}

Series gas chromatography-mass spectrometry (GC-MS) is a traditional method for volatile substances analysis, but the instrument is bulky and expensive. A common alternative is to use solid-state gas sensors that rely on variations in conductivity, capacitance, work function, mass, optics, and reaction energy that may depend on gas-solid interactions.

\subsection{Temperature as A Biomarker}

Temperature is closely related to inflammation and infection at the wound site, and abnormal changes in wound temperature can be an early predictor of infection before other obvious symptoms appear[40]. An inflammatory response caused by bacterial infection causes the wound to overheat, usually $1-2^{\circ} \mathrm{C}$ higher than normal skin. So far, a variety of temperature measuring tools have been developed, including infrared thermometers, colorimetric sensors and electronic temperature sensors[41-42]. Qian Pang et al., combined with the development of emerging bioelectronics, studied an intelligent flexible electronic integrated wound dressing, which is expected to provide early diagnosis of infection through the real-time monitoring of wound temperature by integrated sensors, and provide on-demand infection treatment through the release of antibiotics. The integrated system has good flexibility, good compatibility, high monitoring sensitivity and durability. This proof-of-concept study holds great promise for developing new strategies to significantly improve wound management and other pathological diagnosis and treatment[43]. Min Gong has proposed a flexible respiratory electronic device with real-time temperature sensing and the ability to deliver wound anti-infection treatment on demand in a timely manner. The conductive polymer nanoomentum has good flexibility, reliable air permeability and good environmental stability. In addition, the flexible temperature sensor can be combined with a wireless transmitter to realize real-time wireless temperature monitoring[44].

\section{3 pH as A Biomarker}

The $\mathrm{pH}$ value of wound solution affects wound healing and is an important biomarker of wound state. It can detect $\mathrm{pH}$ changes within the physiologically relevant range with high accuracy and precision, and can insight into the change of wound state in a convenient and non-invasive way. At present, the wound $\mathrm{pH}$ measurement mainly includes electrochemical sensor, $\mathrm{pH}$ electrode and $\mathrm{pH}$ test paper. Additional optical sensor layers for detecting wound $\mathrm{pH}$ have been proposed and have shown that wound healing is accompanied by a 
continuous decrease in $\mathrm{pH}$. Literature[45]proposed a preparation method of optical sensor material that combines indicator dye and inert dye to make indicator dye change from green to red[45]. Yingnan $\mathrm{Zhu}$ et al. developed a multifunctional zwitterionic gel wound dressing as an optical sensor for simultaneous monitoring of $\mathrm{pH}$ and glucose levels in diabetic wound treatment. Phenol red showed obvious color changes in the relative $\mathrm{pH}$ range (4-8) during wound healing. The visible images were collected by smart phones and converted into RGB signals to quantify wound parameters. The changes of glucose and $\mathrm{pH}$ of wound surface in diabetic mice were monitored[46]. The $\mathrm{pH}$ value of the wound bed is an important parameter because it reflects and influences many physiological and biochemical processes that occur during wound remodeling. In chronic and infected wounds, the $\mathrm{pH}$ is usually alkaline between 7.5 and 8.9 [47]. The normal $\mathrm{pH}$ of the skin is slightly acidic (5.5-6), but elevated $\mathrm{pH}$ is found in the initial stages of both acute and chronic wounds. However, acute wounds quickly return to a normal acidic $\mathrm{pH}$, while chronic wounds remain alkaline $(7.4+)$ for several months.

\subsection{Wound Moisture as A Biomarker}

Wound moisture is a key parameter to ensure the optimal wound healing condition, and moisture balance is very important to achieve the optimal wound healing condition. Optimal moisture balance is important as wet wounds can lead to maceration, too little moisture can dry the wound, and too much or too little moisture can delay wound healing. Effective management of wound moisture can shorten wound healing time and dressing change times, so as to reduce nursing time and improve patient comfort. David McColl et al. proposed a new measurement system that monitors moisture content in real time based on sensors placed at the wound/dressing interface. Using a moisture sensor reduces the number of dressing changes and allows the clinician to determine the most appropriate type of dressing needed to provide the best healing conditions for the wound[48].Current signs of excessive moisture or breakage on a dressing are usually visual changes in the material or leakage of the dressing, and it is difficult to tell if there is too little moisture or if there is a damp condition without removing the dressing and interfering with the healing process.

Stephen D Milne et al.[49] studied the measurement of wound moisture content in routine operations to observe the moisture status of the wound during dressing change. The study is also the first large-scale observational study to investigate wound moisture during dressing change. The results show that when the moisture content reading is in the best moisture content range, the moisture content is $44.9 \%$. Of the 30 patients recruited for this study, 11 had an optimal moisture reading of at least $50 \%$ prior to dressing change, suggesting that the protocol currently followed can be modified to allow for fewer dressing changes and less interference with the healing wound bed.

\subsection{Biofilm as A Biomarker}

There is growing evidence that the dominant lifestyle of bacteria in infected wounds is biofilm: a colony of fused bacteria attached to an extracellular polymeric matrix. When a wound forms, it is quickly colonized by bacteria from the surrounding skin environment, including a range of pathogenic bacteria. In many cases, the microbial population of the wound usually remains at a consistently low level of background colonization after the initial infection. There are no obvious barriers to healing and no treatment is required. As the bacterial population continues to expand to the point where the bacterial load exceeds the host's immune clearance, tissue invasion is allowed, triggering clinically relevant infections. Strict criteria for the identification of biofilm-related diseases have not been clearly established. This makes it difficult to translate basic science biofilm studies in vitro into clinical Settings for wound infection.

N. T. Thet et al. investigated a wound dressing made of hydrated agarose film in contact with pathogenic wound biofilms[50]. The understanding of biofilm phenotypes is important for the understanding of bacteria in vitro, but applying biofilm science to the clinic has been difficult. Stephen C. Davis et al. created a biofilm-associated wound model. The results show that this model is helpful to elucidate the role of bacterial biofilm in wound infection and healing. It will also provide a tool to study antibacterial action in complex wound[51]. In the existing methods, fluorescent dye-labeled polysaccharides connected with lectin or dye-labeled proteins connected with specific sequences are used to indicate the structure and content of extracellular matrix polymers, as well as automatic color-changing intelligent dressing and photothermal effect combined with surface-enhanced Raman scattering imaging technology.

\section{Discussion}

In this review, we highlight the current status of clinically applicable biomarkers. Biomarkers have traditionally been considered an important area of medical research: the measurement of some biomarkers contributes to a better understanding of pathophysiology, while others are used to assess the effectiveness of specific treatments or for prognostic purposes.

Bacterial metabolites as markers of wound infection usually require costly and time-consuming sample preparation. Current techniques for monitoring bacterial metabolites include capillary zone electrophoresis, real-time polymerase chain reaction next-generation sequencing, fluorescence in situ hybridization/confocal laser scanning microscopy, metal oxide semiconductor sensors, etc. When inflammation occurs in the body, the level of inflammatory factors is increased and lasts for a long time, so it can be used to assist the early diagnosis of acute infection and evaluate the severity of infection. Current monitoring techniques for cytokines and inflammatory factors include biological activity detection, immunological detection, molecular biological detection, etc. When a wound becomes infected, a variety of bacterial metabolic enzymes promote tissue breakdown, prolonging inflammation. For enzyme detection, the method of antigen antibody is often used, which has the characteristics of strong specificity and high efficiency. At present, the detection technology of enzyme includes surface plasmon resonance imaging, electrochemical immune sensor, nano sensor and so on. Temperature is a marker of rapid succession. When a wound is infected, the body's immune response and the 
increase in vasodilation and tissue metabolism induced by inflammatory cytokines lead to a rise in temperature, which can be considered as a sign of wound infection. At present, the techniques for detecting wound infection temperature include thermal responsive polymer nanofiber dressing and infrared thermometer. The measurement of $\mathrm{PH}$ value is helpful for early detection of local infection or severe bacterial fixed value, so as to carry out early treatment intervention on wound surface. Considering that there may be too little or too much wound exudation or interference of the exudation itself (bloody, suppurative wound, etc.) in clinical use, the clinical promotion of this kind of response dressing is still in progress. Current techniques for wound $\mathrm{PH}$ detection include two-dimensional luminescence based on time-domain luminescence imaging and PH sensors. The moisture balance in the wound is important because wet wounds can lead to maceration, too little moisture can dry the wound, and too much or too little moisture can delay wound healing. Effective management of wound moisture can shorten healing time and dressing change times, thus reducing nursing time and improving patient comfort. Although it has been demonstrated that wound infection affects normal healing of both acute and chronic wounds[52], the role of bacterial biofilms in wound healing and skin infections remains unclear. They are encased in self-generated and host-generated extracellular polymer matrices that adhere to living or abiotic surfaces[53].

Biomarkers can be classified as predictive, diagnostic and indicative according to the type of information they provide. Predictive biomarkers can be used to predict outcomes or provide the likelihood of therapeutic benefit. They can serve as a powerful tool for customizing treatment patterns for specific patient populations. Diagnostic biomarkers can be used to identify the presence of single or multiple factors that may influence clinical outcomes. Indicative biomarkers can be used to monitor disease progression or response to treatment in real time. It is hoped that this biomarker could be personalized for assessment and provide predictions of a patient's healing status and insights into treatments. The pathophysiology of wound infection is very complex. Proper treatment of infected wound areas and limiting the occurrence of organ dysfunction, which is actually a key determinant of morbidity and mortality, are critical. In this context, the search for biomarkers could play an important role.

\section{Conclusions and Prospects}

Many potential biomarkers are currently being identified using different sample collection approaches and molecular methods. A focus on simplicity and consistent implementation of these biomarkers, as well as an emphasis on effective follow-up, is essential to translate this technology into clinically viable point-of-care applications. Rapid and appropriate diagnosis of wound infection is an indispensable task in wound management. The development of diagnostic tools to monitor wound status online will help trained personnel select appropriate treatment as early as possible. Improved diagnostic tools to enable early prevention of infection will bring great benefits to individuals and society[54]. Biofilms seem to be an interesting biomarker because bacteria in biofilms are a constant in wounds, and detection of wound biofilms directly reflects infection. They will play a relevant role in rapid and simple wound monitoring to determine whether a wound is infected. Other infection biomarkers, such as bacteria and their metabolites, represent promising indicators. However, wound infection cannot be determined by the unique concentration and pathogenicity of wound contaminants alone, but is more complex. Odor sensing method for wound infection detection has the advantages of no invasion, quick response, economical and convenient use, but it also has some disadvantages such as poor anti-interference ability and difficult quantitative analysis. Taste sensing directly measures infection markers in wounds. This method has strong explanatory ability and the ability of quantitative analysis of infection markers. However, it is invasive and requires professional dyes and primers, so it is difficult to work for a long time. Physical sensing method is convenient to use and can quickly detect wounds without invasion. However, because it does not directly detect the biochemical process of wounds, it is difficult to realize the early detection of wound infection and the identification of wound pathogenic microorganisms. The sensor of wound infection information is the basis of wound infection detection. Only through effective analysis can the detection target be finally achieved. Clinical evaluation of novel diagnostic devices is critical in terms of effective treatment and poses a major challenge in the near future.

\section{References}

[1] Eaglstein W H, Falanga V. Chronic wounds[J]. Surgical Clinics of North America, 1997, 77(3): 689-700.

[2] St Ad Elmann W K, Digenis A G, Tobin G R. Impediments to wound healing $[\mathrm{J}]$. American Journal of Surgery, 1998, 176(2A Suppl): 39S.

[3] Knighton D R, Ciresi K F, Fiegel V D, et al. Classification and treatment of chronic nonhealing wounds. Successful treatment with autologous platelet-derived wound healing factors (PDWHF) $[\mathrm{J}]$. Annals of Surgery, 1986, 204(3): 322-330.

[4] Ippolito E. Epidemiologia e implicanze socio-economiche delle ulcere degli arti inferiori. In: Monti M, editor. Lulcera cutanea: approccio multidisciplinare alla diagnosied al trattamento. Milano: Springer-Verlag Italia[M]. 2000.

[5] Turns, Martin. The diabetic foot: An overview of assessment and complications[J]. British Journal of Nursing, 2011, 20(Sup8): S19.

[6] Gottrup F. A specialized wound-healing center concept: importance of a multidisciplinary department structure and surgical treatment facilities in the treatment of chronic wounds[J]. American Journal of Surgery, 2004, 187(5A): S38-S43.

[7] Crovetti G, Martinelli G, Issi M, et al. Platelet gel for healing cutaneous chronic wounds[J]. Transfusion \& Apheresis Science, 2004, 30(2): 145-151.

[8] Singer A J, Clark R A F. Cutaneous wound healing[J]. New England journal of medicine, 1999, 341(10): 738-746.

[9] Salvo P, Dini V, Di Francesco F, et al. The role of biomedical sensors in wound healing $[\mathrm{J}]$. Wound Medicine, 2015, 8: 15-18.

[10] Medzhitov R. Origin and physiological roles of inflammation[J]. Nature, 2008, 454(7203): 428-435. 
[11] Nathan C. Points of control in inflammation[J]. Nature, 2002(6917): 846-852.

[12] Medzhitov R. Origin and physiological roles of inflammation[J]. Nature, 2008, 454(7203):428-435.

[13] Ludwig J A, Weinstein J N. Biomarkers in cancer staging, prognosis and treatment selection[J]. Nature Reviews Cancer, 2005, 5(11): 845-856.

[14] Ferrari, Mauro. Cancer nanotechnology: opportunities and challenges[J]. Nature Reviews Cancer, 2005, 5(3):161-171.

[15] Kingsmore, Stephen F. Multiplexed protein measurement: technologies and applications of protein and antibody arrays[J]. Nature Reviews Drug Discovery, 2006, 5(4): 310-320.

[16] Liu R, Ye X, Cui T. Recent progress of biomarker detection sensors[J]. Research, 2020, 2020(4):1-26.

[17] Abayomi L A, Terry L A, White S F, et al. Development of a disposable pyruvate biosensor to determine pungency in onions (Allium cepa L.) $[\mathrm{J}]$. Biosensors \& Bioelectronics, 2006, 21(11): 2176-2179.

[18] Abdelshafi N A, Bell, Jérémy, Rurack K, et al. Microfluidic electrochemical immunosensor for the trace analysis of cocaine in water and body fluids[J]. Drug Testing and Analysis, 2019, 11.

[19] Sean, Goggins, Ellen, et al. Ratiometric electrochemical detection of hydrogen peroxide and glucose[J]. Organic \& Biomolecular Chemistry, 2017.

[20] Rocio D, Miguel O, Chávez Giovanny, et al. The evaluation of a low-cost colorimeter for glucose detection in salivary samples[J]. Sensors, 2017, 17(11):2495-.

[21] Kassal P, Zubak M, Scheipl G, et al. Smart bandage with wireless connectivity for optical monitoring of $\mathrm{pH}[\mathrm{J}]$. Sensors and actuators. B, Chemical, 2017, B246(JUL.): 455-460.

[22] Deng W J, Wang L F, Dong L, et al. LC wireless sensitive pressure sensors with microstructured PDMS dielectric layers for wound monitoring[J]. IEEE Sensors Journal, 2018, (99): 1-1.

[23] Emma Daulton, Alfian Wicaksono, Janak Bechar, James A. Covington, Joseph Hardwicke. The detection of wound infection by ion mobility chemical analysis[J]. Biosensors, 2020, 10(3).

[24] Cooksey E M, Singh G, Scott L C, et al. Detection of coliphages and human adenoviruses in a subtropical estuarine lake-science direct[J]. Science of The Total Environment, 2019, 649:1514-1521.

[25] Xu T, Li J, Zhang S, et al. Integration of diagnosis and treatment in the detection and kill of Saureus in the whole blood[J]. Biosensors \& Bioelectronics, 2019, 142:111507.

[26] Allardyce R A, Langford V S, Hill A L, et al. Detection of volatile metabolites produced by bacterial growth in blood culture media by selected ion flow tube mass spectrometry (SIFT-MS)[J]. Journal of Microbiological Methods, 2006, 65(2): 361-365.

[27] Bailey A, Pisanelli A M, Persaud K C. Development of conducting polymer sensor arrays for wound monitoring[J]. Sensors \& Actuators B Chemical, 2008, 131(1):5-9.

[28] Gregor, Tegl, Doris, et al. Biomarkers for infection: enzymes, microbes, and metabolites[J]. Applied Microbiology \& Biotechnology, 2015.
[29] Korotcentcov G. Metal oxides for solid state gas sensors. What determines our choice?[J]. Materials Science and Engineering, 2008, 61:1-39.

[30] Xu Xun-tao. Research on key technology of medical electronic nose[D]. Chongqing University, 2009.

[31] Wang JUN, Cui Shaoqing, Chen Xinwei, et al. Research progress of electronic nose sensor technology and application[J]. Transactions of the Chinese Society for Agricultural Machinery, 2013.

[32] Thomas A N, Riazanskaia S, Cheung W, et al. Novel noninvasive identification of biomarkers by analytical profiling of chronic wounds using volatile organic compounds[J]. Wound Repair and Regeneration, 2010, 18(4):391-400.

[33] Jingwei Feng, Tian F, Yan J, et al. A background elimination method based on wavelet transform in wound infection detection by electronic nose $[\mathrm{J}]$. Sensors and Actuators B: Chemical, 2011.

[34] Berna A Z, Anderson A R, Trowell S C. Bio-Benchmarking of Electronic Nose Sensors[J]. PLoS ONE, 2009, 4(7): e6406.

[35] Xu Ruishan. Application value of procalcitonin in clinical severe bacterial infection[J]. Journal of Clinical Laboratory Testing (electronic version), 2018, 007(003): 568-568.

[36] Chen Tangtian, Wu Xiannan, Li Mengmeng, et al. Advances in host biomarkers for differentiating bacterial and viral infections[J]. Medical Review 202026 volumes 16 pages 3135-3140, ISTIC, 2020.

[37] Blokhuis-Arkes M, Haalboom M, Job V, et al. Rapid enzyme analysis as a diagnostic tool for wound infection: Comparison between clinical judgment, microbiological analysis, and enzyme analysis[J]. Wound Repair \& Regeneration, 2015, 23(3):345-352.

[38] Andrea, Hasmann, Ulrike, Gewessler, Elisabeth, Hulla, Konstantin P, Schneider, Barbara, Binder, Antonio, Francesko, Tzanko, Tzanov, Michael, Schintler, Job, Van der Palen, Georg M, Guebitz, Eva, Wehrschuetz-Sigl. Sensor materials for the detection of human neutrophil elastase and cathepsin G activity in wound fluid[J]. Experimental Dermatology, 2011, 20(6):508-513.

[39] Dargaville T R, Farrugia B L, Broadbent J A, et al. Sensors and imaging for wound healing: A review $[\mathrm{J}]$. Biosensors \& Bioelectronics, 2013, 41(none):30-42.

[40] Fierheller M, Sibbald R G. A clinical investigation into the relationship between increased periwound skin temperature and local wound infection in patients with chronic leg ulcers[J]. Advances in Skin \& Wound Care, 2010, 23(8):369.

[41] Tang N, Zheng Y, Jiang X, et al. Wearable sensors and systems for wound healing-related $\mathrm{pH}$ and temperature detection[J]. Micromachines, 2021, 12(4):430.

[42] Lahiri B B, Bagavathiappan S, Jayakumar T, et al. Medical applications of infrared thermography: A review[J]. Infrared Physics \& Technology, 2012, 55(4):221-235.

[43] Pang Q, Lou D, Li S, et al. Smart flexible electronics-integrated wound dressing for real-time monitoring and on-demand treatment of infected wounds[J]. Advanced Science, 2020.

[44] Gong M, Wan P, Ma D, et al. Flexible breathable nanomesh electronic devices for on demand therapy[J]. 
Advanced Functional Materials, 2019, 29(26): 1902127.1-1902127.11.

[45] MOHR G J, MüLLER H. Tailoring colour changes of optical sensor materials by combining indicator and inert dyes and their use in sensor layers, textiles and non-wovens[J]. Sens \& Actuators B: Chemical, 2015, 206: 788-793.

[46] Zhu Y, Zhang J, Song J, et al. A multifunctional pro-healing zwitterionic hydrogel for simultaneous optical monitoring of $\mathrm{pH}$ and glucose in diabetic wound treatment $[\mathrm{J}]$. Advanced Functional Materials, 2020, 30(6): 1905493.1-1905493.9.

[47] Mclister A, Mchugh J, Cundell J, et al. New developments in smart bandage technologies for wound diagnostics[J]. Advanced Materials, 2016: 5732-5737.

[48] D Mccoll, Cartlidge B, Connolly P. Real-time monitoring of moisture levels in wound dressings in vitro: An experimental study[J]. International Journal of Surgery, 2007, 5(5): 316-322.

[49] Milne S D, Seoudi I, Hamad H A, et al. A wearable wound moisture sensor as an indicator for wound dressing change: an observational study of wound moisture and status[J]. International Wound Journal, 2016, 13(6):1309-1314.

[50] Thet N T, Alves D R, Bean J E, et al. Prototype development of the intelligent hydrogel wound dressing and its efficacy in the detection of model pathogenic wound biofilms[J]. Acs Appl Mater Interfaces, 2016, 8(24): 14909-14919.

[51] Davis S C, Ricotti C, Cazzaniga A, et al. Microscopic and physiologic evidence for biofilm-associated wound colonization in vivo[J]. Wound Repair and Regeneration, 2010, 16.

[52] Robson, M. C. Wound infection. A failure of wound healing caused by an imbalance of bacteria[J]. Journal of Urology, 1998, 159(4): 1414-1415.

[53] Davey, ME, O'toole, et al. Microbial biofilms: from ecology to molecular genetics[J]. MICROBIOL MOL BIOL REV, 2000, 2000, 64(4): 847.

[54] Hartman E, Wallblom K, Plas M, et al. Bioinformatic analysis of the wound peptidome reveals potential biomarkers and antimicrobial peptides $[\mathrm{J}]$. Frontiers in Immunology, 2021, 11. 